\title{
LA TUBERCULOSE DANS L'aRMÉE RUSSE
}

La statistique du service de santé de l'armée russe pour l'année 1905 a paru en 1907; nous en trouvons une analyse, due à la plume de M. Fromont 1, dans les Archives de Médecine et de Pharmacie militaires de mai dernier.

Relevons-y cette seule mention qui concerne la tuberculose : "Nombre des cas : $1^{\circ}$ Pulmonaires: 3360 cas $=2,8 \%$ 2276 réformes $=1,900 \% 715$ décès $=0,59 \% \%$. 20 Autres organes : 1630 cas $=1,40 \% ; 1207$ réformes $=1 \% \%$; 113 décès $=0,09 \%$ Le cercle le moins éprouvé est celui de la Sibérie; le plus éprouvé celui de Saint-Pétersbourg, $9,1 \% \%$. Dans ce dernier cercle, certains régiments ont eu jusqu'à $30,1 \% \%$ de leur effectif atteint (grenadiers); le régiment Preobrajenski, le moins éprouvé, a encore de ce fait 15,2 ${ }^{00} \%$ de morbidité. Le Comité technique, se basant sur le rapport des médecins de corps, déclare que cette énorme morbidité provient de l'existence, à l'arrivée des recrues, de tuberculoses latentes que ne peuvent déceler les méthodes cliniques ordinaires et de l'impossibilité d'envoyer dans des sanatoria inexistants les hommes fatigués ou soupconnés de tuberculose. ”

Cette citation confirme les conclusions de notre rapport sur * la tuberculose dans les armées ), à la Conférence de Londres. Le service militaire est un générateur de tuberculose, surtout pendant la première année du service, parce que les fatigues qu'il exige de jeunes hommes encore peu résistants, à l'âge où l'éclosion des formes thoraciques de cette maladie est la plus fréquente' font apparaitre des états jusque là latents, chez les prédisposés et les débiles. En refoulant ces victimes du service militaire dans la population civile, l'armée contribue puissamment à la diffusion de la tuberculose dans la population.

Toute organisation sanitaire militaire consciente de ses responsabilités visera donc aussi bien à serrer de très près la question des visites sanitaires aux recrutements qu'à entrer en rapport avec l'aulorité civile pour que les tuberculeux qui quiltent l'armée soient l'objet d'une surveillance suffisante, aussi bien dans leur intérèt

\footnotetext{
1 Revue médicale des armées, p. 370.
} 
que dans celui de la population civile dans laquelle ils rentrent. Jusqu'ici rien de semblable ne s'est fait dans la plupart des armées. Les rapports sanitaires de l'armée russe constatent aujourd'hui celte lacune pour ce qui la concerne; les lignes que nous a vons citées plus haut en font foi. Il nous est donc permis d'espérer que cette question si urgente y sera mise à l'étude à nouveau. Les méthodes cliniques permettant de déceler les états tuberculeux ou prétuberculeux ne sont plus aussi insuffisantes, comme le dit le rapport russe, que ci-devant et les mesures prophylactiques, recommandées par les ligues antituberculeuses et réalisées plus ou moins dans quelques Etals, dans des villes et dans des groupements sociaux ou industriels, ont prouvé que nous ne sommes point désarmés contre l'extension de ce mal, loin de là. On est donc en droit d'attendre des autorités sanitaires des armées qu'elles participent, elles aussi, dans l'intérêt même de l'armée, aux efforts qui sont poursuivis de loute part par la campagne anti-tuberculeuse, et nous souhaitons que la Croix-Rouge, lien normal entre les services sanitaires des armées et les autorités sanitaires civiles, puisse intervenir efficacement dans cette campagne bienfaisante. Cette participation à une des plus grandes nuvres de solidarité humaine constituerait, pour la Croix-Rouge, une de ses plus utiles fonctions en temps de paix.

$D^{r}$ Ferribie.

\section{SAXE}

\section{LA SOCIÉté SAXONNE EN 1907 ET $19 ! 8$}

La Société, tout en laissant encore certaines tâches en arrière, s'est développée cependant et enrichie au cours de ces deux dernières années de façon à pouvoir remplir sa fonction principale, celle d'auxiliaire efficace du service sanitaire officiel, pour l'accom. plissement des tâches qui lui incomberaient au début d'une guerre. 